Research Article

\title{
Using Galileo and BDS-3 Quad-Frequency Signals for Long-Baseline Instantaneous Decimeter-Level Positioning
}

\author{
Wang Gao $\mathbb{D}^{1,2}$ Liwei Liu $\mathbb{D D}^{1}{ }^{1}$ Longlei Qiao $\mathbb{D}^{3},{ }^{3}$ and Shuguo Pan $\mathbb{D}^{1,2}$ \\ ${ }^{1}$ School of Instrument Science and Engineering, Southeast University, Nanjing 210096, China \\ ${ }^{2}$ Key Laboratory of Micro-Inertial Instrument and Advanced Navigation Technology, Southeast University, Nanjing 210096, China \\ ${ }^{3}$ Nanjing Compass Navigation Technology Company Limited, Nanjing 210008, China
}

Correspondence should be addressed to Wang Gao; gaow@seu.edu.cn

Received 7 May 2021; Revised 28 May 2021; Accepted 7 June 2021; Published 15 June 2021

Academic Editor: Chao $\mathrm{Hu}$

Copyright (C) 2021 Wang Gao et al. This is an open access article distributed under the Creative Commons Attribution License, which permits unrestricted use, distribution, and reproduction in any medium, provided the original work is properly cited.

As the signals of Galileo and the global BDS-3 navigation satellite system have been accessible, positioning users can use quadfrequency even five-frequency signals nowadays. With multifrequency signals, one can form some useful combinations to improve the positioning performance, e.g., the widely used extra-wide-lane (EWL)/wide-lane (WL) in triple-frequency cases. For quad-frequency or five-frequency cases, better positioning performance can be expected since additional frequencies are introduced. In this study, we systematically analyse the benefits of Galileo and BDS-3 quad-frequency signals on long-baseline instantaneous positioning. First, the theoretical analysis of EWL/WL ambiguity resolution (AR) and satellite-station range estimation with a single-satellite geometry-free and ionosphere-free model is studied, along with the comparison with triplefrequency cases. Second, using the quad-frequency advantages, an instantaneous decimeter-level positioning model is proposed, where the geometry-free model is adopted for the first two EWL AR and the geometry-based model is adopted for the third WL AR. In the end, the AR and positioning performance are evaluated using real long-baseline date containing Galileo and BDS-3 quad-frequency observations. The results indicate that, with quad-frequency observations, both Galileo and BDS-3 EWL/WL ambiguities can be fixed reliably with a single epoch. Contributed by the resolved EWL/WL ambiguities, instantaneous decimeter-level positioning can be obtained, with the accuracies of $0.116 \mathrm{~m} / 0.126 \mathrm{~m} / 0.351 \mathrm{~m}$ in north, east, and up directions, respectively.

\section{Introduction}

Chinese BDS-3 has started to provide full operational capability services by July 31, 2020. Along with GPS, GLONASS, and Galileo, many more satellites and frequencies are becoming available. At present (September 21, 2020), there are 22 usable Galileo satellites and 29 usable BDS- 3 satellites in orbit. All these satellites can transmit more than three frequencies for positioning, navigation, and timing (PNT) service, where Galileo transmits signals on five frequencies centered at E1 $(1575.42 \mathrm{MHz})$, E5a $(1176.45 \mathrm{MHz}), \mathrm{E} 5 \mathrm{~b}$ $(1207.14 \mathrm{MHz})$, E5 $(1191.795 \mathrm{MHz})$, and E6 $(1278.75 \mathrm{MHz})$ [1], and BDS- 3 transmits signals on five frequencies centered at B1C (1575.42 MHz), B1I (1561.098 MHz), B2a (1176.45 $\mathrm{MHz}), \mathrm{B} 2 \mathrm{~b}(1207.14 \mathrm{MHz})$, and B3I (1268.52 MHz) [2].
With the availability of signals from more frequencies, the performance of real-time kinematic (RTK) is expected to be further improved, since more observation redundancies are introduced and more possible combinations can be formed $[3,4]$.

Researches about using multifrequency GNSS have been started since the late 1990s, when Forssell et al. [5] and Vollath et al. [6] first proposed the classical three-frequency ambiguity resolution (TCAR) method. Enge et al. [7] and Hatch et al. [8] proposed the cascaded integer resolution (CIR) method, which also adopted the stepwise geometryfree $(\mathrm{GF})$ rounding strategy like TCAR. After that, many researchers further proposed some modified triple-frequency AR or positioning model. Compared with the early GF models, Vollath [9], Feng and Rizos [10], Hatch [11], 
Feng and Li [12], and Tang et al. [13] applied the geometrybased (GB) model to multiple-carrier AR as well, which can further enhanced the AR model strength. Generally, whether the GF or GB model, the basic and also the core idea of these models is using the advantages of some extra-wide-lane (EWL)/wide-lane (WL) combinations, which usually has the characteristics of long wavelength, week ionosphere effect, or relative low noise [14]. With these triple-frequency models, cycle slip detection and repair, ambiguity resolution, and positioning accuracy have been verified to be significantly improved.

Among the numerous advantages of multifrequency GNSS signals, a notable advantage is that some EWL/WL ambiguities can be resolved more easily, even with only a single epoch in some cases. Single-epoch AR essentially support instantaneous positioning, which will be very meaningful for some applications, e.g., precise vehicle navigation and self-driving. In these application scenarios, users prefer the faster (even instantaneous), more continuous, and more reliable positioning and navigation performance $[15,16]$. Although short-baseline instantaneous centimeter-level RTK positioning with multisystem observations is possible, it is limited by the short-baseline length. Network-RTK enables large-scale positioning situation; however, it needs dense reference stations, which is usually unavailable for some special cases, e.g., offshore positioning. For medium or long baselines, instantaneous narrow-lane (NL) AR has always been difficult due to the short wavelength and easily affected by the unmodelled errors, even though multifrequency observations are applied [17]. Another rapid precise positioning approach is using ambiguityresolved EWL/WL carrier phase observations, which can be seen as more precise pseudoranges $[15,18]$. With triplefrequency simulated data, Li et al. [18] and Feng and Li [19] proposed the long-range RTK positioning model, and instantaneous submeter- to meter-level positioning could be obtained. If the amplified noise could be smoothed with NL carrier phase multiple epochs, the positioning results would be more precise. Later with real triple-frequency observations gradually available from BDS-2, GPS, and Galileo, the positioning performance using ambiguity-resolved EWL/ WL carrier phase observations is verified [20-22]. In recent two years, Laurichesse and Banville [23] and Geng et al. $[24,25]$ also using ambiguity-resolved EWL/WL in undifferenced precise point positioning (PPP) to obtain rapid positioning results.

Nowadays, Galileo and BDS-3 signals with more than three frequencies have been feasible for most survey-grade receivers. It is necessary to reveal the benefits of these multifrequency signals on PNT services. In this study, we will develop the combined Galileo and BDS-3 quad-frequency signals, i.e., Galileo E1, E5a, E5b, and E6 and BDS-3 B1C, $\mathrm{B} 1 \mathrm{I}, \mathrm{B} 2 \mathrm{a}$, and B3I signals, on long-baseline instantaneous positioning. The rest of this paper is organized as follows: in Section 2, the basic observation equations for quad-frequency combinations are introduced, together with the stochastic model for the observation equations. In Section 3, theoretical analysis of quad-frequency EWL/WL AR and range estimation is studied, and an instantaneous decimeterlevel positioning model is proposed. In Section 4, we mainly test the single-epoch $\mathrm{AR}$ and instantaneous positioning performance using real long-baseline quad-frequency data. Some conclusions will be given in Section 5 .

\section{Materials and Methods}

2.1. Fundamental Equations and Corresponding Stochastic Model. Without loss of simplicity, double-difference (DD) carrier phase and pseudorange observation equations in meters can be described as follows:

$$
\left\{\begin{array}{l}
\Delta \phi_{j}=\Delta \rho+\Delta T-\eta_{j} \Delta I_{1}+\lambda_{j} \Delta N_{j}+\Delta \varepsilon_{\phi_{j}} \\
\Delta P_{j}=\Delta \rho+\Delta T+\eta_{j} \Delta I_{1}+\Delta \varepsilon_{P_{j}}
\end{array}\right.
$$

where $\Delta$ is the station- and satellite-difference double-difference operator; $\phi$ is the carrier phase observation and $p$ is pseudorange observation; $\rho$ represents geometric distance from satellite to receiver; $T$ is the troposphere delay; $I_{1}$ is the first-order ionosphere delay on the first frequency; $\eta_{j}=f_{1}^{2} / f_{j}^{2}$ represents the ionosphere scale factor on $j$ th frequency; $f_{j}$ is signal frequency; $\lambda_{j}$ is the wavelength at the corresponding frequency; $N_{j}$ is the integer phase ambiguity; $\varepsilon_{\phi_{j}}$ and $\varepsilon_{P_{j}}$ represent the noise for carrier phase and pseudorange measurement, respectively.

With quad-frequency signals for Galileo or BDS-3, the carrier phase and code linear observation combinations can be formulated as

$$
\left\{\begin{aligned}
\Delta \phi_{(i, j, k, m)}= & \frac{i \cdot f_{1} \cdot \Delta \phi_{1}+j \cdot f_{2} \cdot \Delta \phi_{2}+k \cdot f_{3} \cdot \Delta \phi_{3}+m \cdot f_{4} \cdot \Delta \phi_{4}}{i \cdot f_{1}+j \cdot f_{2}+k \cdot f_{3}+m \cdot f_{4}} \\
\Delta P_{(i, j, k, m)} & =\frac{i \cdot f_{1} \cdot \Delta P_{1}+j \cdot f_{2} \cdot \Delta P_{2}+k \cdot f_{3} \cdot \Delta P_{3}+m \cdot f_{4} \cdot \Delta P_{4}}{i \cdot f_{1}+j \cdot f_{2}+k \cdot f_{3}+m \cdot f_{4}}
\end{aligned}\right.
$$


where the subscript $i, j, k, m$ are integer coefficients of combinations. For the combined observations, the corresponding $f_{(i, j, k, m)}, \quad \lambda_{(i, j, k, m)}, \quad \eta_{(i, j, k, m)}, \quad N_{(i, j, k, m)}$ can be expressed as

$$
\left\{\begin{array}{l}
f_{(i, j, k, m)}=i \cdot f_{1}+j \cdot f_{2}+k \cdot f_{3}+m \cdot f_{4}, \\
\lambda_{(i, j, k, m)}=\frac{c}{f_{(i, j, k, m)}}, \\
\eta_{(i, j, k, m)}=\frac{f_{1}^{2}\left(i / f_{1}+j / f_{2}+k / f_{3}+m / f_{4}\right)}{f_{(i, j, k, m)}} \\
\Delta N_{(i, j, k, m)}=i \cdot \Delta N_{1}+j \cdot \Delta N_{2}+k \cdot \Delta N_{3}+m \cdot \Delta N_{4} .
\end{array}\right.
$$

Theoretically, $i, j, k, m$ could be arbitrary integers, so that an infinite number of combinations can be formed. According to the existing researches, the sum of those observation combinations with long wavelength, week ionosphere effect, and low noise is equal to 0 . In the study, we mainly focus on the instantaneous positioning based on single-epoch AR; thus only those carrier phase EWL/WL combinations with $i+j+k+m=0$ are considered. According to the previous study in $[20,22]$, only $n-1$ independent EWL/WL combinations can be formed for $n$ frequencies. Then for the purpose of instantaneous positioning calculation, the carrier phase observations in (1) can be replaced with three independent EWL/WL combinations, as in the following equation:

$$
\left\{\begin{array}{l}
\Delta \phi_{\left(i_{1}, j_{1}, k_{1}, m_{1}\right)}=\Delta \rho+\Delta T-\eta_{\left(i_{1}, j_{1}, k_{1}, m_{1}\right)} \Delta I+\lambda_{\left(i_{1}, j_{1}, k_{1}, m_{1}\right)} \Delta N_{\left(i_{1}, j_{1}, k_{1}, m_{1}\right)}+\Delta \varepsilon_{\phi_{\left(i_{1}, j_{1}, k_{1}, m_{1}\right)}}, \\
\Delta \phi_{\left(i_{2}, j_{2}, k_{2}, m_{2}\right)}=\Delta \rho+\Delta T-\eta_{\left(i_{2}, j_{2}, k_{2}, m_{2}\right)} \Delta I+\lambda_{\left(i_{2}, j_{2}, k_{2}, m_{2}\right)} \Delta N_{\left(i_{2}, j_{2}, k_{2}, m_{2}\right)}+\Delta \varepsilon_{\phi_{\left(i_{2}, j_{2}, k_{2}, m_{2}\right)}}, \\
\Delta \phi_{\left(i_{3}, j_{3}, k_{3}, m_{3}\right)}=\Delta \rho+\Delta T-\eta_{\left(i_{3}, j_{3}, k_{3}, m_{3}\right)} \Delta I+\lambda_{\left(i_{3}, j_{3}, k_{3}, m_{3}\right)} \Delta N_{\left(i_{3}, j_{3}, k_{3}, m_{3}\right)}+\Delta \varepsilon_{\phi_{\left(i_{3}, j_{3}, k_{3}, m_{3}\right)}} .
\end{array}\right.
$$

Although the selected three EWL/WL combinations in (4) are independent, the cross correlations still need to be properly handled in the stochastic model, as the basic carrier phase measurements are crossly used in these combinations. In addition, when forming the difference among satellites, the correlation between the reference satellite and nonreference satellites also needs to be considered. Considering the two kinds of correlations, the stochastic model matrix $\mathbf{R}$ for the uncombined pseudorange observations and the combined carrier-phase observations can be expressed as

$$
\mathbf{R}=\left[\begin{array}{ll}
\mathbf{R}_{\Delta P} & \\
& \mathbf{R}_{\Delta \phi}
\end{array}\right]=\left[\begin{array}{ll}
\mathbf{D}_{1} \mathbf{R}_{\Delta P, 0} \mathbf{D}_{1}^{T} & \\
& \mathbf{D}_{3}\left(\mathbf{D}_{2} \mathbf{R}_{\Delta \phi, 0} \mathbf{D}_{2}^{T}\right) \mathbf{D}_{3}^{T}
\end{array}\right],
$$

where $\mathbf{R}_{\Delta P}$ and $\mathbf{R}_{\Delta \phi}$ represent the pseudorange part and carrier phase part, respectively. $\mathbf{R}_{\Delta P, 0}$ and $\mathbf{R}_{\Delta \phi, 0}$ represent the stochastic model matrix of independent station-differenced (SD) pseudorange and carrier phase observations, respectively, as

$$
\left\{\begin{array}{l}
\mathbf{R}_{\Delta P, 0}=\operatorname{diag}\left(\left[\sigma_{\Delta P_{1,1}}^{2}, \sigma_{\Delta P_{2,1}}^{2}, \sigma_{\Delta P_{3,1}}^{2}, \sigma_{\Delta P_{4,1}}^{2}, \ldots, \sigma_{\Delta P_{1, n}}^{2}, \sigma_{\Delta P_{2, n}}^{2}, \sigma_{\Delta P_{3, n}}^{2}, \sigma_{\Delta P_{4, n}}^{2}\right]\right), \\
\mathbf{R}_{\Delta \phi, 0}=\operatorname{diag}\left(\left[\sigma_{\Delta \phi_{1,1}}^{2}, \sigma_{\Delta \phi_{2,1}}^{2}, \sigma_{\Delta \phi_{3,1}}^{2}, \sigma_{\Delta \phi_{4,1}}^{2}, \ldots, \sigma_{\Delta \phi_{1, n}}^{2}, \sigma_{\Delta \phi_{2, n}}^{2}, \sigma_{\Delta \phi_{3, n}}^{2}, \sigma_{\Delta \phi_{4, n}}^{2}\right]\right) .
\end{array}\right.
$$

In (6), $\sigma_{\Delta P_{i, n}}^{2}$ and $\sigma_{\Delta \phi_{j, n}}^{2}(j=1,2,3,4)$ represent the variances of $\mathrm{SD}^{1, n}$ pseudorange and carrier phase, respectively, where the second subscript $1, \ldots, n$ refers to the satellite index. Further, $\sigma_{\Delta P_{j, n}}^{2}$ and $\sigma_{\Delta \phi_{j, n}}^{2}$ can be derived based on the exponential elevation weighting function [26] and SD amplification. Empirically, we set the a priori zenith-referenced phase and code precision (standard deviation) for undifferenced observations empirically to $2 \mathrm{~mm}$ and $30 \mathrm{~cm}$, respectively, in this study. The satellitedifference operation matrix $\mathbf{D}_{1}$ for pseudorange observations, the cross correlation operation matrix $\mathbf{D}_{2}$, and the satellite-difference operation matrix $\mathbf{D}_{3}$ for combined carrier phase observations can be expressed as in the following equations: 


$$
\begin{aligned}
& \mathbf{D}_{1}=\left[\begin{array}{ll}
-\mathbf{I}_{4 \times 4} \otimes \mathbf{e}_{n-1} & \mathbf{I}_{4 \times 4} \otimes \mathbf{I}_{(n-1) \times(n-1)}
\end{array}\right], \\
& \mathbf{D}_{2}=\left[\begin{array}{cccc}
\frac{i_{1} \cdot f_{1}}{f_{\left(i_{1}, j_{1}, k_{1}, m_{1}\right)}} \frac{j_{1} \cdot f_{2}}{f_{\left(i_{1}, j_{1}, k_{1}, m_{1}\right)}} \frac{k_{1} \cdot f_{3}}{f_{\left(i_{1}, j_{1}, k_{1}, m_{1}\right)}} \frac{m_{1} \cdot f_{4}}{f_{\left(i_{1}, j_{1}, k_{1}, m_{1}\right)}} \\
\frac{i_{2} \cdot f_{1}}{f_{\left(i_{2}, j_{2}, k_{2}, m_{2}\right)}} \frac{j_{2} \cdot f_{2}}{f_{\left(i_{2}, j_{2}, k_{2}, m_{2}\right)}} \frac{k_{2} \cdot f_{3}}{f_{\left(i_{2}, j_{2}, k_{2}, m_{2}\right)}} \frac{m_{2} \cdot f_{4}}{f_{\left(i_{2}, j_{2}, k_{2}, m_{2}\right)}} \\
\frac{i_{3} \cdot f_{1}}{f_{\left(i_{3}, j_{3}, k_{3}, m_{3}\right)}} \frac{j_{3} \cdot f_{2}}{f_{\left(i_{3}, j_{3}, k_{3}, m_{3}\right)}} \frac{k_{3} \cdot f_{3}}{f_{\left(i_{3}, j_{3}, k_{3}, m_{3}\right)}} \frac{m_{3} \cdot f_{4}}{f_{\left(i_{3}, j_{3}, k_{3}, m_{3}\right)}}
\end{array}\right] \otimes \mathbf{I}_{n \times n}, \\
& \mathbf{D}_{3}=\left[\begin{array}{ll}
-\mathbf{I}_{3 \times 3} \otimes \mathbf{e}_{n-1} & \mathbf{I}_{3 \times 3} \otimes \mathbf{I}_{(n-1) \times(n-1)}
\end{array}\right] .
\end{aligned}
$$

In order to avoid the intersystem biases [27, 28], Galileo and BDS-3 observations are processed with the conventional intrasystem model; i.e., no intersystem differencing is formed.

\subsection{Theoretical Analysis of EWL/WL Ambiguity Fixing and Its} Benefits on Range Estimation. In (4), one has many choices for selecting three independent EWL/WL combinations. However, for the purpose of reliable AR and positioning estimation, we need to find the optimal choices. For the aspect of positioning, actually arbitrary three independent ambiguity-resolved EWL/WL combinations will lead to the same results [22]. Then we only need to be concerned with the EWL/WL selection for AR performance. A reasonable criterion is that the EWL/WL AR should have maximal success rate, i.e., the minimum variance. However, it is complicated to determine the three combinations simultaneously through rigorous theoretical derivation. Here, we adopt the strategy proposed by Laurichesse and Banville [23], where a sequential determination method is used with considering the constraint of the fixed ambiguities to unfixed ambiguities. In their method, a simple geometry-free and ionosphere-free (GIF) model with estimation of the DD satellite-station ranges is adopted, so that the quantized ambiguity and range precision can be derived from the parameter variances. For each DD satellite-pair, combining the four uncombined pseudorange observations and the first EWL combination with undetermined coefficients, we simultaneously estimate three parameters, i.e., the comprehensive DD satellite-station range which contains $\Delta \rho, \Delta T$, and other geometric items, the DD ionosphere delay $\Delta I$, and the EWL ambiguity. The alternative integer coefficients $i, j, k, m$ are selected within $[-10,10]$. During the adjustment calculation, the DD pseudorange and carrier phase precision are set as $0.5 \mathrm{~m}$ and $5 \mathrm{~mm}$, respectively. In these alternative combinations, we can choose the candidate with minimum ambiguity variance as the first EWL combination. Referring to the frequency order of Galileo E1-E5a-E5b-E6 and BDS-3 B1C-B1I-B2a-B3I, Galileo $(0,-1,1,0)$ and BDS-3 $(1,-1,0,0)$ are the best in all combinations, with the ambiguity precision of 0.043 cycles and 0.041 cycles, respectively (shown in Table 1). According to the formula for rounding success rate in (10) [29], the high-precision float ambiguities can be reliably fixed with a priori success rate of almost $100 \%$.

$$
P(-0.5<x<0.5)=\int_{-0.5}^{0.5} \frac{1}{\sigma \sqrt{2 \pi}} \exp \left(-\frac{x^{2}}{2 \sigma^{2}}\right) 0 x .
$$

When the first EWL ambiguity is fixed, the ambiguityresolved EWL observation can be treated as pseudorange to assist the following ambiguity estimation. The second optimal EWL/WL combinations are not unique, as there are several combinations that have the same minimum variances with 0.060 cycles and 0.110 cycles, respectively, in each system. For simplicity, in these optimal combinations we select the ones with small coefficients as the representatives, which are $(0,0,-1,1)$ for both Galileo and BDS-3. Overall, the second EWL ambiguities can also be fixed reliably with high a priori success rate larger than 99.9\%. Meanwhile, from Table 1 we can see that even though the second EWL ambiguity is resolved directly without the constraint from the first fixed EWL ambiguity, it can also be fixed with high success rate. Particularly for BDS-3, there was almost no difference with or without the constraint, although more decimals are not shown. This means that the first and the second EWL ambiguities can be resolved simultaneously instead of being resolved sequentially. This can slightly simplify the sequential calculation process.

In Table 1, the corresponding range precision is also calculated under the constraint with different number of fixed EWL/WL ambiguities. From Table 1, we can see after fixing two EWL ambiguities that the range precision can be slightly improved from $1.249 \mathrm{~m}$ to $1.169 \mathrm{~m}$ for Galileo and from $1.012 \mathrm{~m}$ to $0.816 \mathrm{~m}$ for BDS-3. However, this improvement is not significant and still cannot meet the demand of decimeter-level positioning. Thus the third EWL or WL ambiguity further needs to be resolved. With the constraint of the two ambiguity-resolved EWL observations, all the third independent EWL or WL combinations will have the same precision as well as the range precision, although not all results are listed here. From the table, we can see Galileo has the ambiguity precision of 0.298 cycles and BDS-3 has the precision of 0.209 cycles, which correspond to the rounding success rates of $90.7 \%$ and $98.3 \%$, respectively. Unlike the first two EWL ambiguities, it cannot be fixed to an integer reliably by rounding directly. Here, we adopt the 
TABLE 1: Ambiguity and range estimation precision for quad-frequency Galileo and BDS-3.

\begin{tabular}{|c|c|c|c|c|c|}
\hline \multirow{2}{*}{ Parameters } & \multirow{2}{*}{ Wavelength (m) } & \multicolumn{4}{|c|}{ Number of fixed EWL/WL ambiguities } \\
\hline & & 0 & 1 & 2 & 3 \\
\hline \multicolumn{6}{|l|}{ Galileo } \\
\hline E5a-E5b: $(0,-1,1,0) /$ cycles & 9.77 & 0.043 & - & - & - \\
\hline E5b-E6: $(0,0,-1,1) /$ cycles & 4.19 & 0.070 & 0.060 & - & - \\
\hline E1-E5a: $(1,-1,0,0) /$ cycles & 0.75 & 0.379 & 0.358 & 0.298 & - \\
\hline Range/m & - & 1.249 & 1.173 & 1.169 & 0.316 \\
\hline \multicolumn{6}{|l|}{$B D S-3$} \\
\hline B1C-B1I: $(1,-1,0,0) /$ cycles & 20.93 & 0.041 & - & - & - \\
\hline B2a-B3I: $(0,0,-1,1) /$ cycles & 3.26 & 0.110 & 0.110 & - & - \\
\hline B1I-B2a: $(0,1,-1,0) /$ cycles & 0.78 & 0.323 & 0.314 & 0.209 & - \\
\hline Range/m & - & 1.012 & 0.949 & 0.816 & 0.333 \\
\hline
\end{tabular}

geometry-based model which is a function of baseline parameters to enhance the model strength. If the third WL ambiguity can also be fixed successfully, it is worth noting that the range estimation can get a remarkable precision, as $0.316 \mathrm{~m}$ for Galileo and $0.333 \mathrm{~m}$ for BDS-3, and thus support decimeter-level positioning in theory.

To reveal the benefits of Galileo and BDS-3 quad-frequency signals compared with the existing triple-frequency GPS and BDS-2, the EWL/WL ambiguity and corresponding range estimation precision in triple-frequency case are listed in $\mathrm{Ta}$ ble 2. Through comparison between Tables 1 and 2, we can see the optimal EWL ambiguities in triple-frequency cases can also get a high precision less than 0.1 cycles. For the WL AR, the quad-frequency cases are better than that in triple-frequency cases on the whole, especially in BDS-3. The most significant improvement is range estimation precision, which is from $0.483 \mathrm{~m}$ (GPS) and 0.516 (BDS-2) to $0.316 \mathrm{~m}$ (Galileo) and $0.333 \mathrm{~m}$ (BDS-3), with the improvement over $30 \%$. This is also the benefit of using the fourth frequency signal.

According to the analysis above, we conclude that the first two EWL ambiguities can be fixed reliably with singleepoch GF model whether for Galileo or BDS-3. Although it may get better AR performance using geometry-based model, the computing efficiency is also a factor which should be considered due to the high-dimensional parameters in multifrequency case. Besides, with the GF model, we can easily determine the EWL ambiguity subset by judging the fractional part of ambiguities, so that reliable ambiguity subset can be easily determined. Thus, in this study, we resolved the first two EWL ambiguities with GF model directly. However, for the third WL ambiguity, since the GF model cannot obtain an ideal precision with a single epoch, the GB model is adopted. In general, as the flowchart in Figure 1, the computing efficiency and AR reliability are coordinately taken into account.

\section{Results and Discussion}

3.1. Data Description and Processing Strategy. In this section, we mainly test the single-epoch EWL/WL AR and instantaneous positioning performance using real Galileo and BDS-3 data. The data of a baseline with the length of $104.2 \mathrm{~km}$ were collected on May 9, 2020, using the Trimble Alloy receiver in Shanxi Province, China. The data collection lasted for 24 hours with the sampling interval of $10 \mathrm{~s}$. Since the instantaneous positioning is emphasized, all the calculation process is carried out with the single-epoch mode. Besides the instantaneous positioning, the troposphere delays are directly corrected with the empirical GPT2w model [30], as if the zenith tropospheric delays (ZTD) or relative zenith tropospheric delay (RZTD) is estimated as unknown parameter(s); it will take a long time for convergence due to the correlation with the height component. Actually, with the advanced empirical model, the relative troposphere delays can be correct with over $90 \%$ and just have small residual errors. The DD ionosphere delays are estimated with consideration of the arbitrariness as no effective empirical model like troposphere delays can be used for long baselines.

The sky plots of the Galileo and BDS-3 satellites used in the long-baseline are shown in Figure 2. We can see at present that BDS-3 has better visibility than Galileo in China region, as there are several GEO and IGSO satellites in BDS3 over Asian-Pacific region and also because Galileo is still in construction period. The numbers of Galileo and BDS-3 visible satellites for the baseline with a cut-off elevation of $10^{\circ}$ are given in Figure 3. We can see with combined Galileo and BDS-3 that there are no less than 10 satellites available at all 24-hour time. This means that some specific positioning applications which rely on quad-frequency or five-frequency have been feasible. The corresponding position dilution of precision (PDOP) is shown in Figure 4. With combined Galileo and BDS-3, we can get a good PDOP smaller than 3.0. Similar to the analysis for Figure 2, this also indicates that the Galileo and BDS-3 can support standalone positioning without GPS and other systems.

3.2. EWL AR Performance with Geometry-Free Model. About the EWL/WL ambiguity resolution, we first investigate the single-epoch EWL AR performance. Figures 5 and 6 show the distribution of EWL ambiguity fraction biases and the corresponding statistics for Galileo and BDS-3, respectively. In theory, the fraction biases may not be equal to the true ambiguity bias due to the integer part. However, after the comparison with the postprocessing multiepoch results, all the EWL ambiguities are confirmed to be fixed correctly in this baseline. From Figures 5 and 6, we can see 
TABLE 2: Ambiguity and range estimation precision for triple-frequency GPS and BDS-2.

\begin{tabular}{lccccc}
\hline System & Frequencies & EWL/cycles & WL/cycles & Range/m & Range (pseudorange)/m \\
\hline GPS & L1, L2, L5 & 0.066 & 0.359 & 0.483 & 1.273 \\
BDS-2 & B1I, B2I, B3I & 0.077 & 0.302 & 0.516 & 1.433 \\
\hline
\end{tabular}

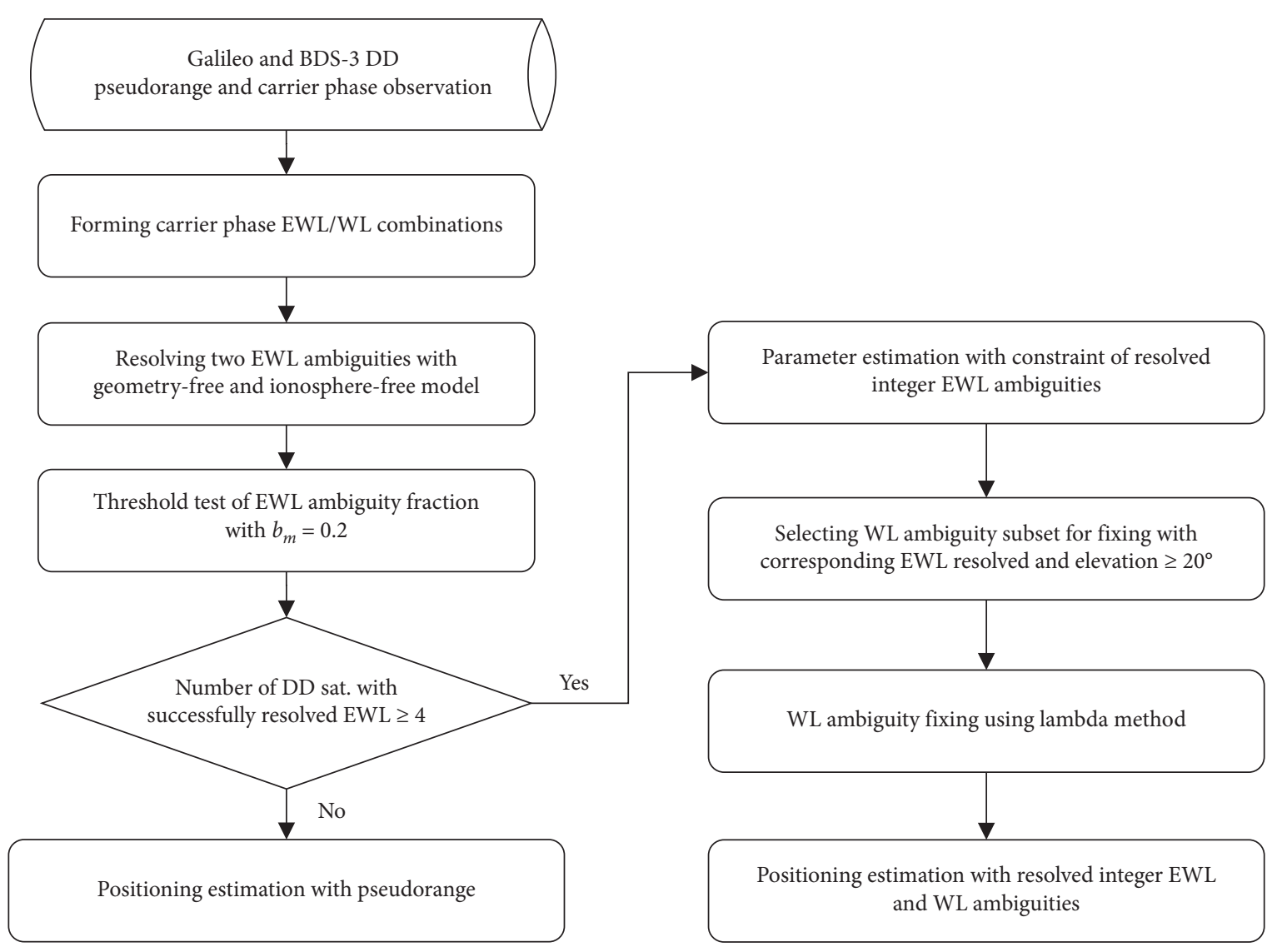

FIgURE 1: The AR and positioning flowchart.

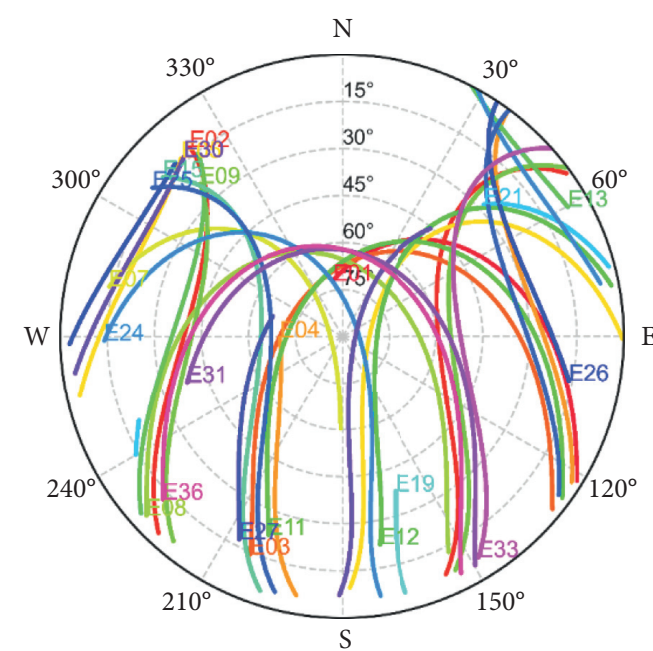

(a)

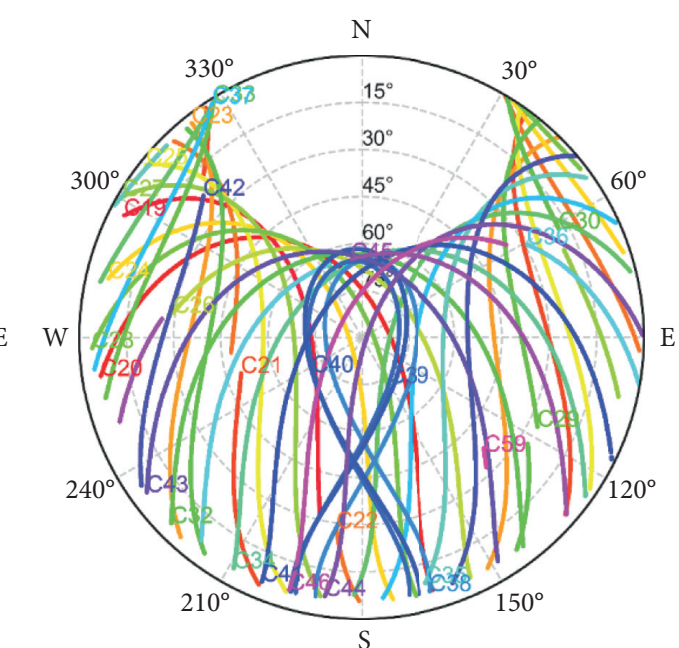

(b)

FIgure 2: The sky plots of Galileo (a) and BDS-3 (b) satellites in the used baseline. 


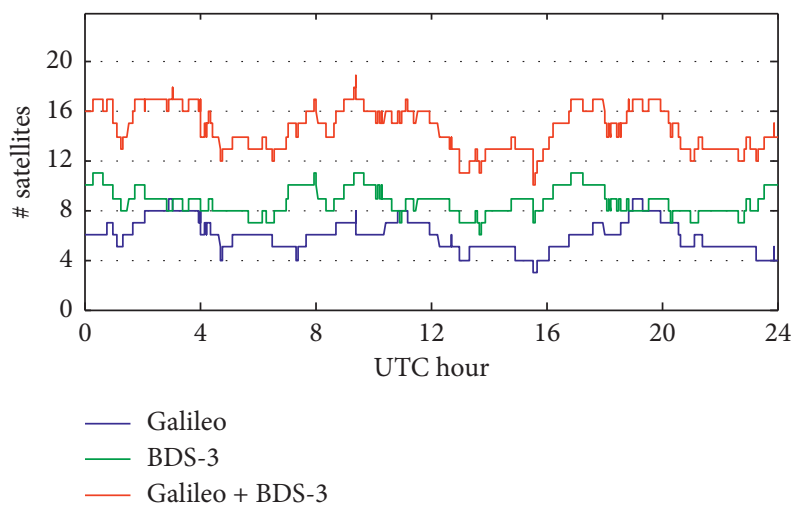

FIGURE 3: Number of Galileo and BDS-3 visible satellites in the used baseline.

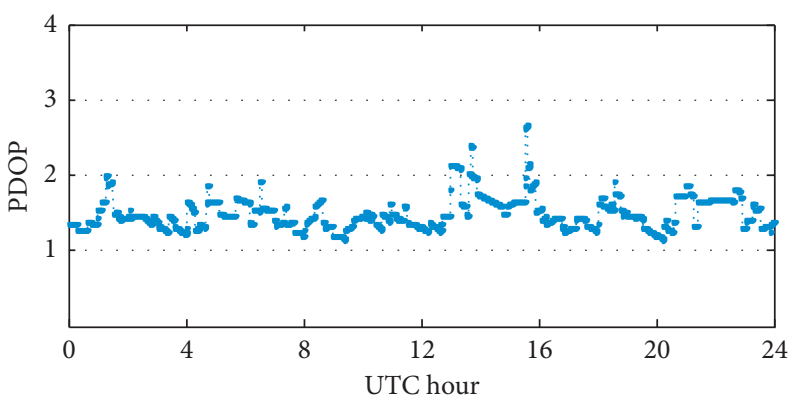

FIGURE 4: PDOP of combined Galileo and BDS-3.

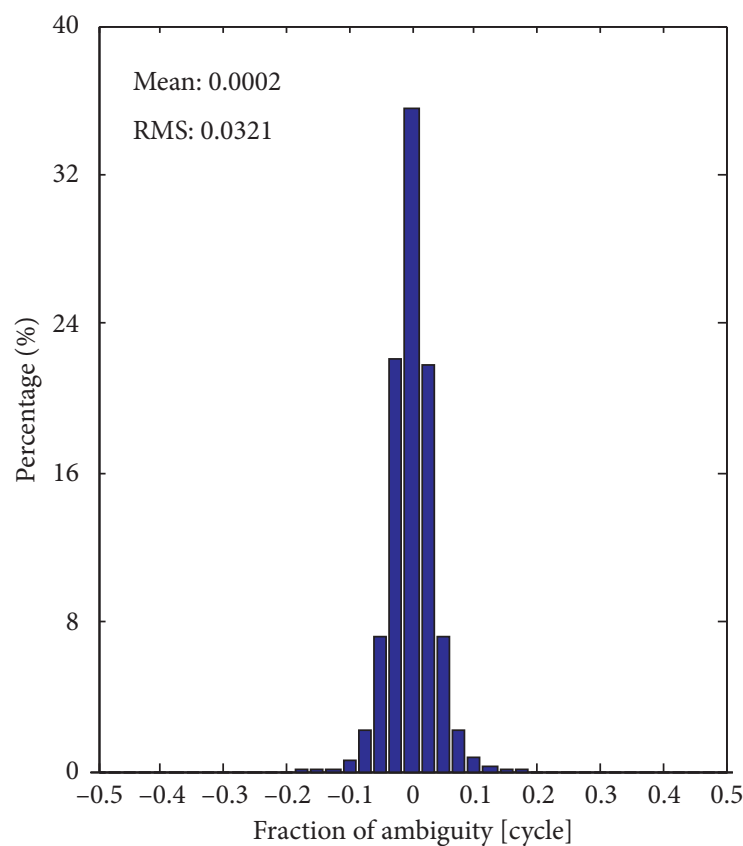

(a)

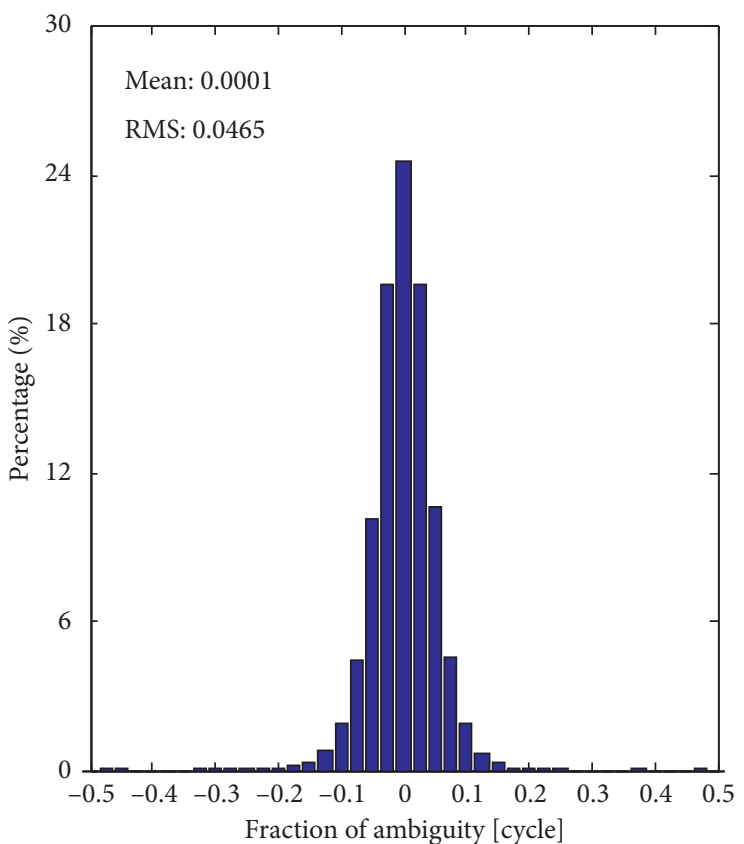

(b)

FIGURE 5: Continued. 


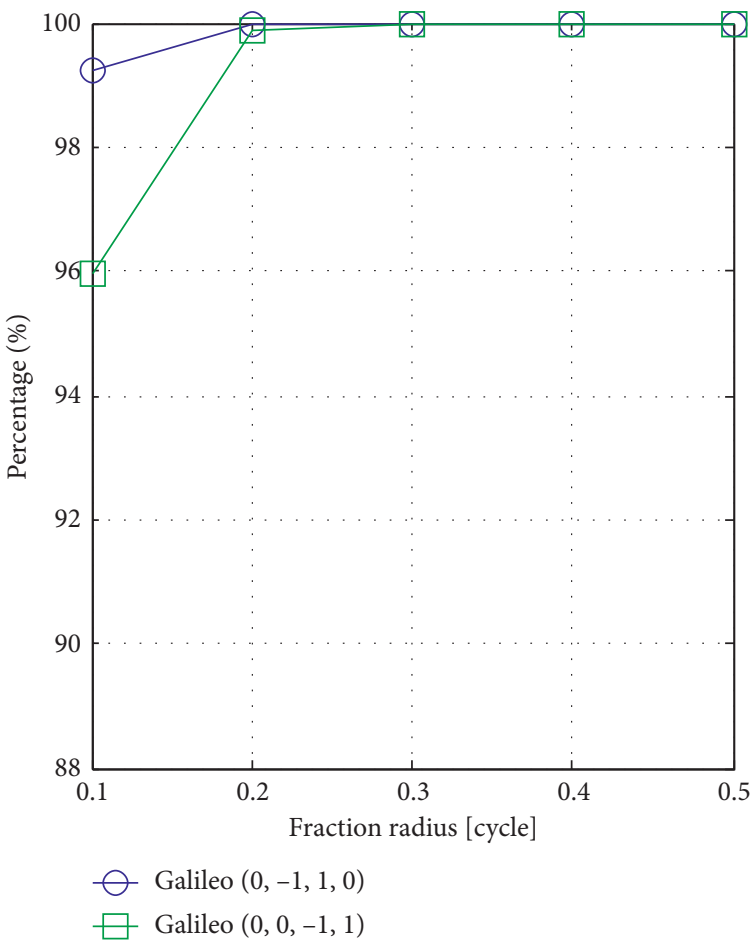

(c)

FIGURE 5: Distribution of Galileo EWL ambiguity fraction biases and the corresponding statistics. (a) Distribution of $(0,-1,1,0)$ fraction biases. (b) Distribution of $(0,0,-1,1)$ fraction biases. (c) Cumulative distribution of EWL fraction biases.

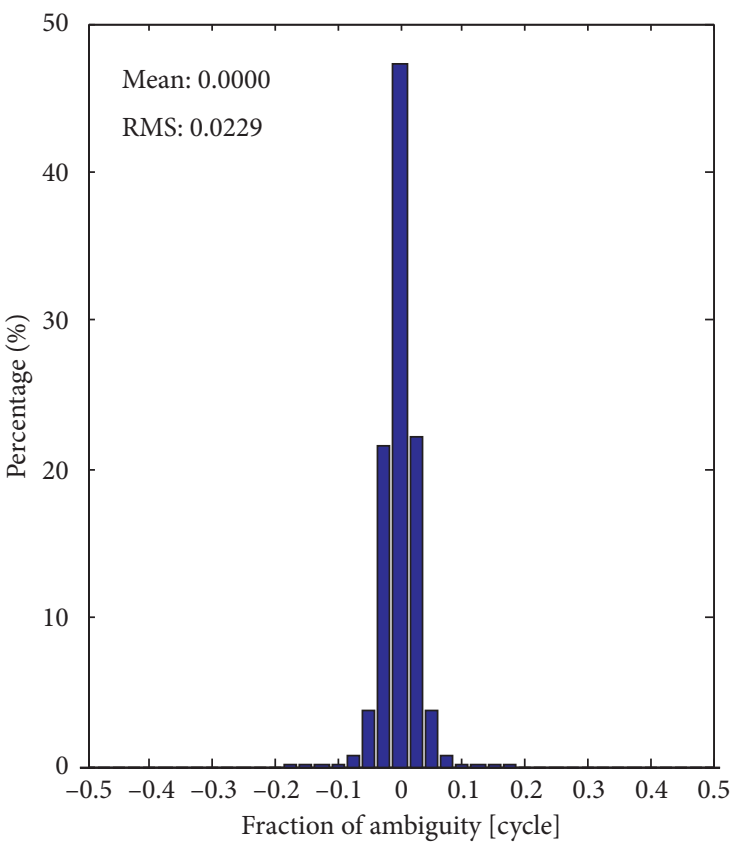

(a)

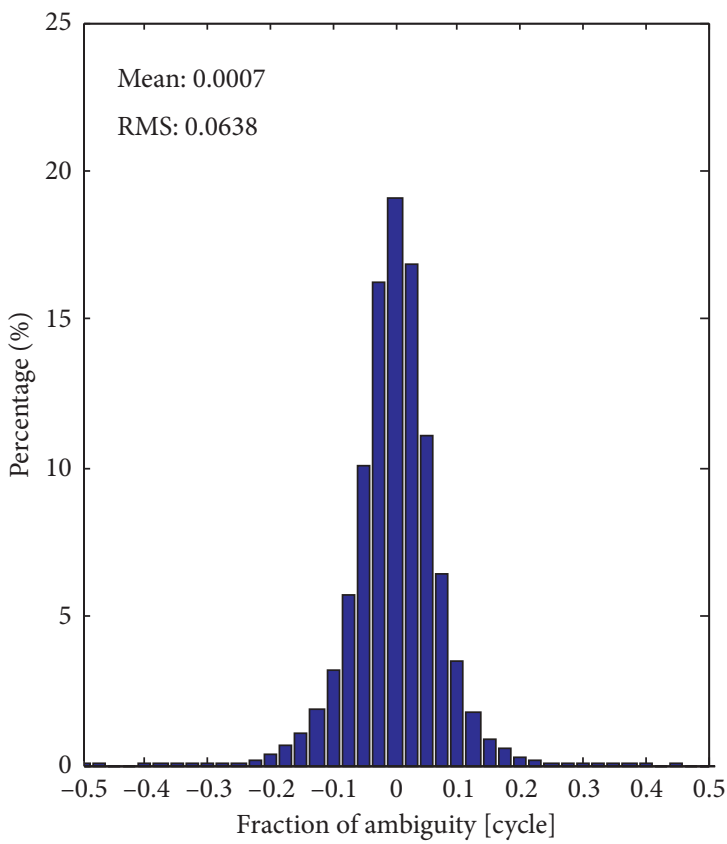

(b)

Figure 6: Continued. 


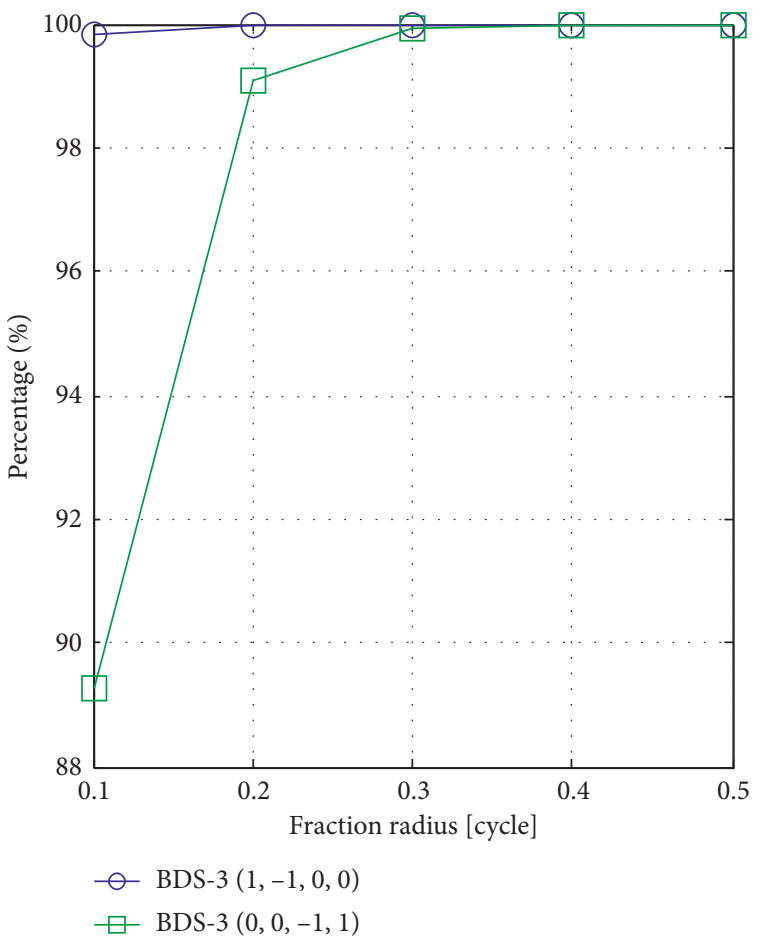

(c)

FIGURE 6: Distribution of BDS-3 EWL ambiguity fraction biases and the corresponding statistics. (a) Distribution of $(1,-1,0,0)$ fraction biases. (b) Distribution of $(0,0,-1,1)$ fraction biases. (c) Cumulative distribution of EWL fraction biases.

with the unbiased GF and IF model that most of the EWL fraction biases are almost within \pm 0.2 cycles and with the mean value of nearly zero. For Galileo, the RMS of the two EWL ambiguity biases are 0.0321 cycles and 0.0465 cycles, while for the BDS-3 they are 0.0229 cycles and 0.0638 cycles. From the high-precision results, we confirm with real data that the first two EWL ambiguities can be resolved very reliably with single epoch. Besides, the EWL AR model is baseline-length independent, since it has to eliminate the troposphere error, ionosphere error, and orbital errors, etc. In practical use, we can further control the AR reliability by checking the fraction bias with a set threshold. In this study, referring to the research in $[31,32]$, the fraction threshold for rounding is set as 0.2 .

3.3. WL AR Performance with Geometry-Based Model. After the EWL ambiguities are resolved, the WL ambiguities can be constrained with the ambiguity-resolved EWL observations as in (4). As analysed previously, the third WL AR with single-epoch GF model cannot get an ideal precision, and thus they are resolved with GB model. In GB model, the baseline components are estimated together with the WL ambiguities, and the residual troposphere errors after correction are still ignored for the instantaneous convergence. The least-squares ambiguity decorrelation adjustment (LAMBDA) method [33] is adopted to fix integer ambiguities due to the correlation among ambiguities. In the WL GB model, the geometric errors are not completely eliminated and the float ambiguities with low elevation are easily

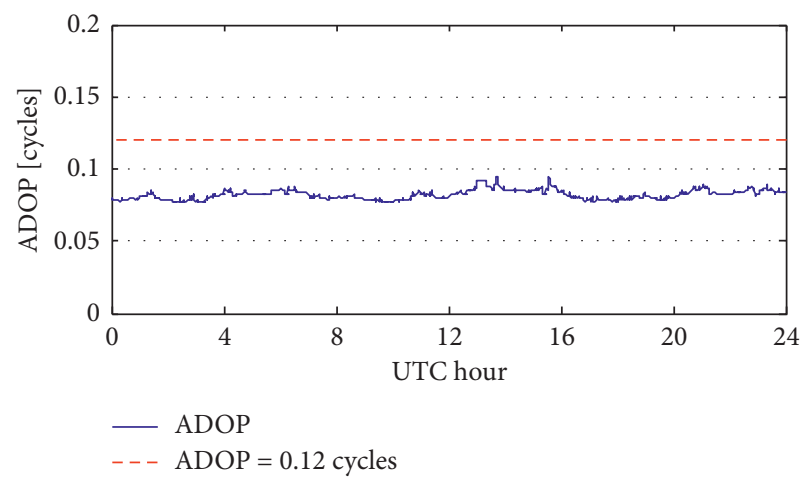

FIGURE 7: ADOP of WL ambiguity resolution with GB model.

affected by the residual geometric errors, measurement noises and multipath effects. Therefore, for the reliable and fixing efficiency, only the ambiguity subset with elevation larger than $20^{\circ}$ is selected to be fixed.

Figure 7 shows the ambiguity dilution of precision (ADOP) series for the WL ambiguity subset, where the red dotted line represents the commonly used threshold of 0.12 cycles, which corresponds to an ambiguity success rate larger than $99.9 \%$. We can see with the GB model and the constraint of EWL observations that the WL ambiguities can be resolved with high a priori success rate. This guarantees the feasibility of model strength for single-epoch ambiguity resolution. Figure 8 shows the ratios of WL AR with LAMBDA, where Figure 8(a) represents the overall ratios and Figure 8(b) represents the ratios 


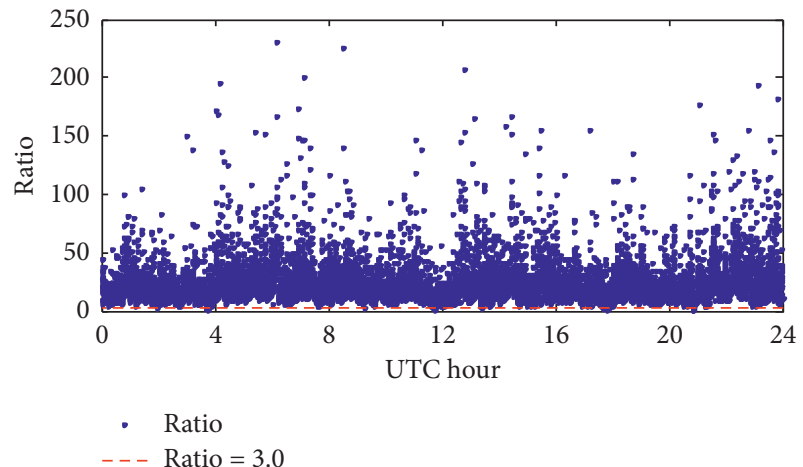

(a)

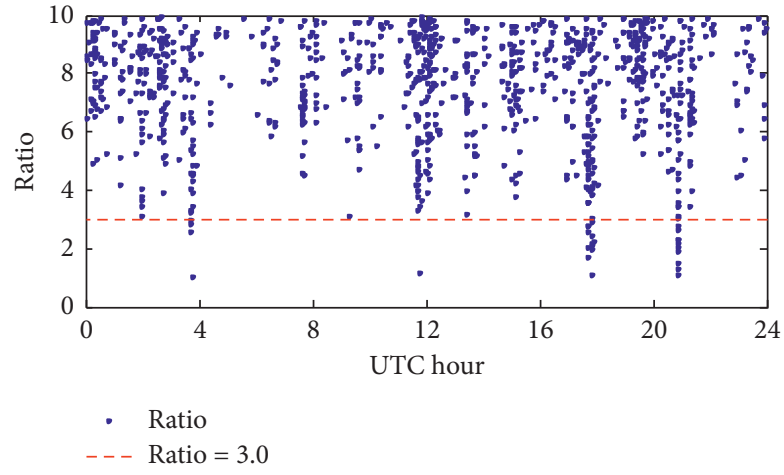

(b)

FIgURE 8: Ratio of WL ambiguity resolution with GB model using LAMBDA method. (a) The overall ratios. (b) The ratios less than 10.
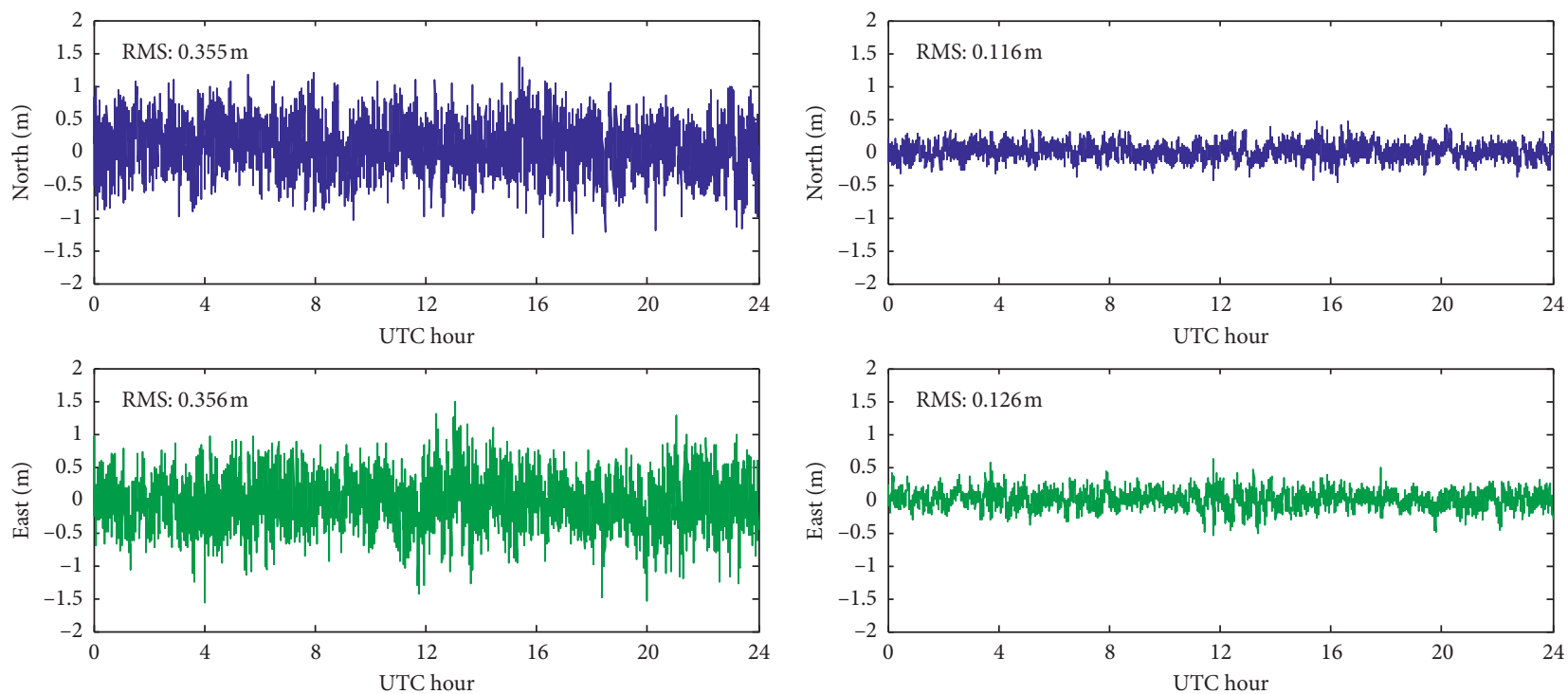

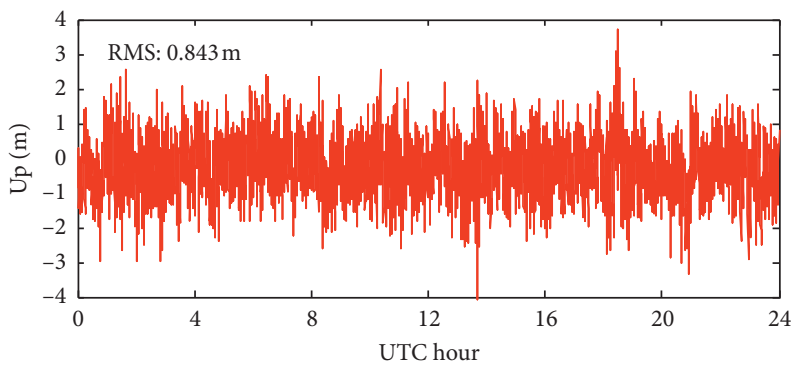

(a)

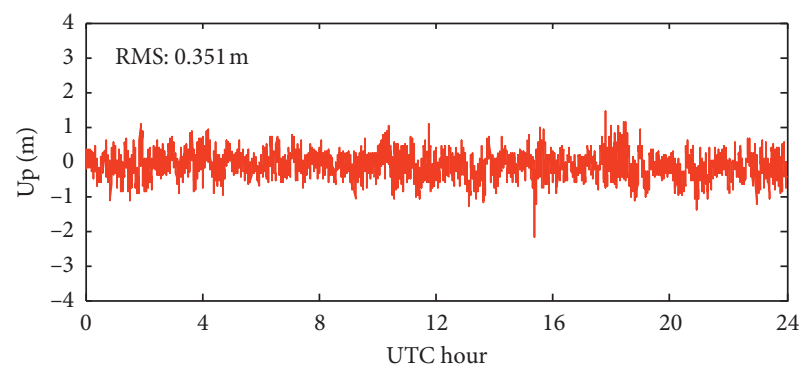

(b)

FIGURE 9: Positioning results with pseudoranges and ambiguity-resolved EWL/WL observations from Galileo and BDS-3 quad-frequency observations. (a) Positioning results with quad-frequency ionosphere-float model of pseudorange observations. (b) Positioning results with quad-frequency ionosphere-float model of EWL/WL observations.

less than 10. The red dotted line represents commonly used ratio threshold of 3.0. In all 8640 epochs, only 27 epochs cannot get ratio values larger than 3.0 , which also means that about 99.7\% epochs can obtain an ideal ambiguity fixing effect. In fact, through comparison with the postprocessing multiepoch $\mathrm{AR}$ results, the WL ambiguities in these 27 epochs are also fixed correctly.
3.4. Instantaneous Positioning Performance with EWL/WL Observations. With three EWL or WL ambiguities resolved, we can implement the instantaneous positioning with the constraint of ambiguity-resolved EWL/WL. Through the analysis before, we know that the satellite-station range precision can be improved to $0.316 \mathrm{~m}$ for Galileo and $0.333 \mathrm{~m}$ for BDS-3; thus the decimeter-level positioning can 
be expected, along with the good PDOP shown in Figure 4. The positioning results are shown in Figure 9. For comparison, the positioning results with quad-frequency ionosphere-float model of pseudorange observations are also shown in Figure 9. As mentioned before, although ratio values in a few epochs cannot reach the threshold of 3.0, the WL ambiguities are also fixed correctly. Thus in Figure 9, the EWL/WL positioning results in all epochs are all produced from ambiguity-fixed solutions. From Figure 9, we see that using pseudoranges, the RMS of positioning errors in three directions are $0.355 \mathrm{~m}, 0.356 \mathrm{~m}$, and $0.843 \mathrm{~m}$, respectively, while with EWL/WL observations, the RMS are $0.116 \mathrm{~m}$, $0.126 \mathrm{~m}$, and $0.351 \mathrm{~m}$. The positioning accuracies in three directions are significantly improved by factors of $3.1,2.8$, and 2.4, respectively. The improvement amplitude is generally consistent compared to the analysis result in Table 1, although a little smaller, which may be the reason that the assumed pseudorange precision relative to carrier phase should be higher. Overall, it can be seen that decimeter-level positioning is verified to be feasible using the quad-frequency EWL/WL observations.

\section{Conclusions}

In this study, we systematically analyse the benefits of Galileo and BDS-3 quad-frequency signals on long-baseline instantaneous decimeter-level positioning. As the research aims at long-baseline positioning, the arbitrariness of ionosphere delays is considered with parameterization and without any ionosphere constraint. The fundamental observation model and stochastic model are introduced, and the theoretical and practical analyses on single-epoch EWL/ WL ambiguity resolution and decimeter-level instantaneous positioning are implemented. Through the theoretical analysis with single-satellite GF and IF model, we reveal that the first two EWL ambiguities whether for Galileo or for BDS-3 can be fixed reliably with high success rate of more than $99.9 \%$. The practical calculation results also verify this conclusion, with unbiased distributions and rather small RMS less than 0.1 cycles. For the third WL AR, since the GF model cannot obtain an ideal precision with single-epoch, the GB model is adopted in this process. With the constraint of EWL observations, the WL AR can get good model strength with ADOP better than 0.1 cycles. In the experiments with real data, over $99.7 \%$ epochs can get ratio values larger than 3.0 and all epochs can get correct ambiguity fixing.

Once the three EWL/WL ambiguities are resolved successfully, one can obtain much more precise range estimation than that using pseudoranges. We also compare the results with the existing triple-frequency GPS and BDS-2. Through comparison, we find that using Galileo and BDS-3 quad-frequency observations, the range estimation precision with ambiguity-resolved EWL/WL observations can be further improved from $0.483 \mathrm{~m}$ (GPS) and $0.516 \mathrm{~m}$ (BDS-2) to $0.316 \mathrm{~m}$ (Galileo) and $0.333 \mathrm{~m}$ (BDS-3). This improvement reflects the most meaningful benefits of Galileo and BDS-3 quad-frequency signals to long-baseline positioning. With the high-precision range estimation, decimeter-level positioning is verified to be feasible, with the RMS of positioning errors of $0.116 \mathrm{~m}, 0.126 \mathrm{~m}$, and $0.351 \mathrm{~m}$ in three directions. It can be expected that this characteristic will also be beneficial for the further estimation of NL ambiguity resolution or other parameters.

It should be noted that the results presented in this study are all calculated with single-epoch mode, as we are mainly concerned with the instantaneous positioning performance. In practical application, as researched in [22], one can use continuous basic carrier phase observations to smooth the noise-amplified EWL/WL observations, and thus better positioning performance can be obtained in subsequent epochs.

\section{Data Availability}

The data used to support the findings of the study are available from the corresponding author upon request.

\section{Conflicts of Interest}

The authors declare that there are no conflicts of interest regarding the publication of this paper.

\section{Acknowledgments}

This work was partially supported by the National Natural Science Foundation of China (grant nos. 41904022 and 41774027) and the Fundamental Research Funds for the Central Universities (grant no. 2242021R41134).

\section{References}

[1] European Union, European GNSS (Galileo) Open Service: Signal in Space: Interface Control document, Publications Office of the European Union, Luxembourg City, Luxembourg, 2010, https://www.gsa.europa.eu/news/galileoopen-service-sis-icd-new-version-12-released.

[2] Y. Yang, Y. Mao, and B. Sun, "Basic performance and future developments of BeiDou global navigation satellite system," Satellite Navigation, vol. 1, no. 1, p. 1, 2020.

[3] K. Wang, A. Khodabandeh, and P. J. G. Teunissen, "Fivefrequency Galileo long-baseline ambiguity resolution with multipath mitigation," GPS Solutions, vol. 22, no. 3, p. 75, 2018.

[4] Z. Zhang, B. Li, X. He et al., "Models, methods and assessment of four-frequency carrier ambiguity resolution for BeiDou-3 observations," GPS Solutions, vol. 24, no. 4, p. 96, 2020.

[5] B. Forssell, M. Martin-Neira, and R. A. Harrisz, "Carrier phase ambiguity resolution in GNSS-2," in Proceedings of the 10th International Technical Meeting of the Satellite Division of the Institute of Navigation (ION GPS 1997), pp. 1727-1736, Kansas City, MO, USA, September 1997.

[6] U. Vollath, S. Birnbach, L. Landau, J. M. Fraile-Ordoñez, and M. Martí-Neira, "Analysis of three-carrier ambiguity resolution technique for precise relative positioning in GNSS-2," Navigation, vol. 46, no. 1, pp. 13-23, 1999.

[7] P. Enge, J. Jung, and B. Pervan, "High integrity carrier phase navigation for future LAAS using multiple civilian GPS signals," IEEE, in Proceedings of the 1999 American Control Conference (Cat. No. 99CH36251), pp. 3650-3654, San Diego, CA, USA, June 1999. 
[8] R. Hatch, J. Jung, P. Enge, and B. Pervan, "Civilian GPS: the benefits of three frequencies," GPS Solutions, vol. 3, no. 4, pp. 1-9, 2000.

[9] U. Vollath, "The factorized multi-carrier ambiguity resolution (FAMCAR) approach for efficient carrier-phase ambiguity estimation," in Proceedings of the 17th International Technical Meeting of the Satellite Division of the Institute of Navigation (ION GNSS 2004), pp. 2499-2508, Long Beach, CA, USA, September 2004

[10] Y. Feng and C. Rizos, "Three carrier approaches for future global, regional and local GNSS positioning services: concepts and performance perspectives," in Proceedings of the 18th International Technical Meeting of the Satellite Division of the Institute of Navigation (ION GNSS 2005), vol. 16, pp. 2277-2278, Long Beach, CA, USA, September 2005.

[11] R. R. Hatch, "A new three-frequency, geometry-free, technique for ambiguity resolution," in Proceedings of the 19th International Technical Meeting of the Satellite Division of the Institute of Navigation (ION GNSS 2006), pp. 309-316, Fort Worth, TX, USA, September 2006.

[12] Y. Feng and B. Li, "A benefit of multiple carrier GNSS signals: regional scale network-based RTK with doubled inter-station distances," Journal of Spatial Science, vol. 53, no. 2, pp. 135-147, 2008.

[13] W. Tang, X. Meng, C. Shi, and J. Liu, “Algorithms for sparse network-based RTK GPS positioning and performance assessment," Journal of Navigation, vol. 66, no. 3, pp. 335-348, 2013.

[14] J. Li, Y. Yang, H. He, and H. Guo, "An analytical study on the carrier-phase linear combinations for triple-frequency GNSS," Journal of Geodesy, vol. 91, no. 2, pp. 151-166, 2017.

[15] B. Li, Y. Shen, and X. Zhang, "Three frequency GNSS navigation prospect demonstrated with semi-simulated data," Advances in Space Research, vol. 51, no. 7, pp. 1175-1185, 2013.

[16] M. Schreiber, C. Knöppel, and U. Franke, "Laneloc: lane marking based localization using highly accurate maps," in Proceedings of the IEEE Intelligent Vehicles Symposium, pp. 449-454, Gold Coast, Australia, June 2013.

[17] P. J. G. Teunissen and N. F. Jonkman, "Will geometry-free full ambiguity resolution be possible at all for long baselines," in Proceedings of the 2001 National Technical Meeting of the Institute of Navigation, pp. 271-280, Long Beach, CA, USA, January, 2001.

[18] B. Li, Y. Shen, and X. Zhang, "Long-range real-time precise navigation with three frequency GNSS," Journal of Wuhan University (Natural Science Edition), vol. 34, no. 7, pp. 782786, 2009.

[19] Y. Feng and B. Li, "Wide area real time kinematic decimetre positioning with multiple carrier GNSS signals," Science China Earth Sciences, vol. 53, no. 5, pp. 731-740, 2010.

[20] B. Li, Y. Feng, W. Gao, and Z. Li, "Real-time kinematic positioning over long baselines using triple-frequency BeiDou signals," IEEE Transactions on Aerospace and Electronic Systems, vol. 51, no. 4, pp. 3254-3269, 2015.

[21] W. Gao, C. Gao, and S. Pan, "Single-epoch navigation performance with real BDS triple-frequency pseudorange and EWL/WL observations," The Journal of Navigation, vol. 69, no. 6, pp. 1293-1309, 2016.

[22] B. Li, Z. Li, Z. Zhang, and Y. Tan, "ERTK: extra-wide-lane RTK of triple-frequency GNSS signals," Journal of Geodesy, vol. 91, no. 9, pp. 1031-1047, 2017.

[23] D. Laurichesse and S. Banville, "Innovation: instantaneous centimeterlevel multi-frequency precise point positioning,"
October 2018, http://www.gpsworld.com/innovationinstantaneous-centimeter-level-multi-frequency-precisepoint-positioning.

[24] J. Geng and J. Guo, "Beyond three frequencies: an extendable model for single-epoch decimeter-level point positioning by exploiting Galileo and BeiDou-3 signals," Journal of Geodesy, vol. 94, no. 1, pp. 1-15, 2020.

[25] J. Geng, J. Guo, H. Chang, and X. Li, "Toward global instantaneous decimeter-level positioning using tightly coupled multi-constellation and multi-frequency GNSS," Journal of Geodesy, vol. 93, no. 7, pp. 977-991, 2019.

[26] H.-J. Eueler and C. C. Goad, "On optimal filtering of GPS dual frequency observations without using orbit information," Bulletin Géodésique, vol. 65, no. 2, pp. 130-143, 1991.

[27] D. Odijk and P. J. G. Teunissen, "Characterization of betweenreceiver GPS-Galileo inter-system biases and their effect on mixed ambiguity resolution," GPS Solutions, vol. 17, no. 4, pp. 521-533, 2013.

[28] J. Paziewski and P. Wielgosz, "Accounting for Galileo-GPS inter-system biases in precise satellite positioning," Journal of Geodesy, vol. 89, no. 1, pp. 81-93, 2015.

[29] P. J. G. Teunissen, "Influence of ambiguity precision on the success rate of GNSS integer ambiguity bootstrapping," Journal of Geodesy, vol. 81, no. 5, pp. 351-358, 2007.

[30] J. Böhm, G. Möller, M. Schindelegger, G. Pain, and R. Weber, "Development of an improved empirical model for slant delays in the troposphere (GPT2w)," GPS Solutions, vol. 19, no. 3, pp. 433-441, 2015.

[31] B. Li, Y. Shen, Y. Feng, W. Gao, and L. Yang, "GNSS ambiguity resolution with controllable failure rate for long baseline network RTK," Journal of Geodesy, vol. 88, no. 2, pp. 99-112, 2014.

[32] W. Gao, C. Gao, S. Pan, X. Meng, and Y. Xia, "Inter-system differencing between GPS and BDS for medium-baseline RTK positioning," Remote Sensing, vol. 9, no. 9, p. 948, 2017.

[33] P. J. G. Teunissen, “The least-squares ambiguity decorrelation adjustment: a method for fast GPS integer ambiguity estimation," Journal of Geodesy, vol. 70, no. 1-2, pp. 65-82, 1995. 